\title{
K-Means with Large and Noisy Constraint Sets
}

\author{
Dan Pelleg and Dorit Baras \\ IBM Haifa Labs \\ dpelleg@il.ibm.com, doritb@il.ibm.com
}

\begin{abstract}
We focus on the problem of clustering with soft instancelevel constraints. Recently, the CVQE algorithm was proposed in this context. It modifies the objective function of traditional $K$-means to include penalties for violated constraints. CVQE was shown to efficiently produce high-quality clustering of UCI data. In this work, we examine the properties of CVQE and propose a modification that results in a more intuitive objective function, with lower computational complexity. We present our extensive experimentation, which provides insight into CVQE and shows that our new variant can dramatically improve clustering quality while reducing run time. We show its superiority in a large-scale surveillance scenario with noisy constraints.
\end{abstract}

\section{Introduction}

Recently, a growing interest in utilizing side-information in clustering led to a variety of new clustering techniques. The side information is used to encode a tacit bias to counter that of the original clustering algorithm. In this sense, the new algorithm can be thought of as supervised. But in contrast to traditional supervision, the ground truth labels need not be explicitly present in the input.

Typically, the side information is in the form of pairwise instance-level constraints. Constraints of this type come in two flavors: a must-link (ML) constraint, to indicate that a pair of input points need to be in the same output cluster, and a cannot-link (CL) constraint, to indicate the opposite. These types of constraints were thoroughly investigated and have been shown to improve results in different application areas. Some of these areas include GPS lane finding [1], video and image indexing [23], robot navigation [4], image segmentation [5], and text categorization 677. The constraints are considered to increase cluster purity, decrease convergence time, and reduce error 8 .

The method in which the constraints are acquired depends on the application itself. For example, spatial or temporal proximity of observations may be used to induce constraints, or user feedback on a clustering result may be used in an active-learning or semi-supervised setting. In experimentation, it is also popular to use the ground truth labels to induce constraints.

In general, existing methods fall into one of two categories: constraint-driven and distance-driven. The first type tries to directly satisfy the constraints. There are hard and soft versions of these, which vary in their ability to ignore some 
constraints. The second type learns a distance metric from the constraints, and it is later used in a constraint-agnostic clustering algorithm.

This work is motivated by a surveillance application. In this setting, a sensor (e.g., a video camera), or a network of such sensors, is located in a public area. The sensor can locate objects in a $2-\mathrm{D}$ or $3-\mathrm{D}$ space. It can also track their movement over a short period of time. For example, if object locations are recorded every minute, the sensor may also identify that the same object that was at location $P$ at time $t$, is at location $P^{\prime}$ at time $t+1$. Noise in the measurements may come in the form of false tracking due to objects or agents leaving and entering the scene, occlusion, etc. The goal of the application is to perform long-term tracking of objects. That is, cluster the observation points such that each cluster corresponds to a single object. This naturally gives rise to the constrained clustering problem with the following characteristics: 1) The number of data points and constraints is large (thousands or more, depending on the monitoring period and frequency). 2) The constraints are mostly ML. 3) The constraints are noisy.

This paper explores solutions to such problems. From the description above, some required properties for such solutions emerge: scalability, efficiency, and resilience to constraint noise. The latter immediately precludes hard satisfaction algorithms. Of the soft variants, algorithms based on $K$-means are a natural fit to the scalability requirement, since they are potentially linear in the number of points, dimensions, constraints and clusters.

We also note the common practice of augmenting the constraint set by transitive and entailed constraints. This is a widely-used heuristic [9], but unfortunately, it cannot be used in our scenario for two reasons. First, the noise may introduce ML constraints between some members of different clusters. When the number of constraints is large, the probability of such an event is high, and the result would be the complete - and useless - clique in the ML graph. Second, the size of the augmented set is huge. In one experiment with around 20000 points and constraints, this kind of pre-processing generated approximately half a million constraints.

Davidson et al. 10 propose using a black-box method to evaluate the usefulness of constraints. Two measurements are defined on constraint sets: informativeness and coherence. Informativeness represents the tacit bias, due to the constraints, that is different from the algorithm's own bias. Coherence is the disparity between ML and CL pairs. These measures can be used to evaluate a given constraint set. In a convincing experiment, an extremely small constraint set is shown to dramatically enhance clustering results. Taking this idea further, the authors suggest using the same measures to filter constraint sets before feeding them to a constrained clustering algorithm. The benefit would be smaller and cleaner sets, resulting in faster operation and increased accuracy. This approach seems like a viable alternative to our scalable algorithms. We look forward to the bridging of the gap between this idea and a working embodiment, enabling us to directly compare the two approaches. 


\section{The CVQE Algorithm}

The unconstrained clustering problem is defined on instances $S_{i}, i=1, \ldots n$ and a parameter $K$ for the number of clusters. Let $C_{j}$ be the centroid representing cluster $j$. Denote by $Q_{j}$ the set of instances that are closest to $C_{j}$. The $K$-means algorithm uses the following update rule: $C_{j}=\frac{1}{\left|Q_{j}\right|} \sum_{s_{i} \in Q_{j}} s_{i}$, where after every centroid update, each instance is reassigned to the cluster of its closest centroid (i.e., the groups $Q_{j}$ are recalculated). This update rule is derived from minimization of the vector quantization error function, $V Q E=\frac{1}{2} \sum_{j=1}^{K} \sum_{s_{i} \in Q_{j}}\left(C_{j}-s_{i}\right)^{2}$.

The Constrained Vector Quantization Error(CVQE) algorithm [4] generalizes $K$-means to handle constraints. It does so by modifying the error function to penalize violated constraints. In the original notation, there are $r$ must-link and $s$ cannot-link constraints, $\left\{\left(s_{1}(i), s_{2}(i)\right)\right\}_{i=1}^{s+r}$. Let $Q_{j}$ be the set of instances assigned to the $j$-th cluster, and $C_{j}$ be the centroid corresponding to the $j$ th cluster. Define $M(x)=\left\{j \mid x \in Q_{j}\right\}$, and let $g(i)=M\left(s_{1}(i)\right)$ and $g^{\prime}(i)=$ $M\left(s_{2}(i)\right)$. Further, let $h(i)$ be the cluster index whose centroid is closest to $C_{i}$. As in Davidson et al. 4, in the case of violation, $s_{2}(i)$ is associated with the violation. Finally, $v(i)$ indicates whether the $i$-th constraint is violated. Namely, for $i=1, \ldots, r, v(i)=1 \leftrightarrow g(i) \neq g^{\prime}(i)$ and for $i=r+1, \ldots, s+r, v(i)=1 \leftrightarrow$ $g(i)=g^{\prime}(i)$, and $v(i)=0$ in all other cases. The update rule is as follows:

$$
C_{j}=\frac{1}{N_{j}}\left\{\sum_{s_{i} \in Q_{j}}\left(s_{i}\right)+\sum_{l=1, g(l)=j}^{r} v(l) \cdot C_{g^{\prime}(l)}+\sum_{l=r+1, g(l)=j}^{s+r} v(l) \cdot C_{h\left(g^{\prime}(l)\right)}\right\}
$$

And $N_{j}=\left|Q_{j}\right|+\sum_{l=1, g(l)=j}^{r+s} v(l)$. Intuitively, in violations of ML constraints, one of the two affected centroids is moved towards the other, wheres CL violations move one of the points towards its next-closest centroid. Similarly to $K$-means, after each iteration, each instance is reassigned to minimize the error function (described below). Hence unlike $K$-means, $Q_{j}$ can contain instances where $C_{j}$ is not their closest centroid. This update rule minimizes the error function $C V Q E=\sum_{j=1}^{K} C V Q E_{j}$, where

$$
C V Q E_{j}=\frac{1}{2} \sum_{s_{i} \in Q_{j}} T_{j, 1}+\frac{1}{2} \sum_{l=1, g(l)=j}^{r} T_{j, 2}+\frac{1}{2} \sum_{l=r+1, g(l)=j}^{r+s} T_{j, 3}
$$

where $T_{j, 1}=\left(C_{j}-s_{i}\right)^{2}, T_{j, 2}=\left[\left(C_{j}-C_{g^{\prime}(l)}\right)^{2} \cdot v(l)\right]$, and $T_{j, 3}=\left[\left(C_{j}-C_{h\left(g^{\prime}(l)\right)}\right)^{2} \cdot v(l)\right]$.

In each step of the CVQE algorithm, each pair of instances that form a constraint are assigned such that the CVQE error function is minimized. The rest of the algorithm (initialization and termination) is the same as $K$-means.

We now discuss some properties of CVQE. First, the order of the points in a constraint is significant. Consider a violated constraint generated by the instances $\left(s_{1}(l), s_{2}(l)\right)$ such that $s_{1}(l) \in Q_{g(l)}, s_{2}(l) \in Q_{g^{\prime}(l)}$. Only $C_{g(l)}$ is affected 


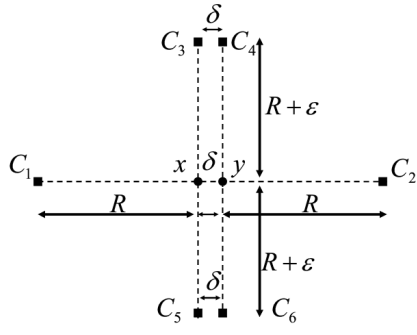

(a)

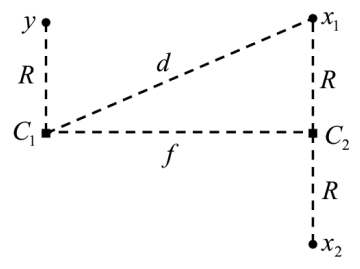

(b)

Fig. 1. CVQE examples

by violation of this link, while $C_{g^{\prime}(l)}$ is not affected at all. This observation holds in both the ML and CL cases.

Second, determining the assignment that minimizes the error function requires $O\left(K^{2}\right)$ calculations for every constraint, which can be expensive when dealing with large numbers of either constraints or clusters. It is also not possible to prune out any possibility (other than the trivial $s_{1}(l) \in Q_{g^{\prime}(l)}, s_{2}(l) \in Q_{g(l)}$ ) from the calculation. To see this, consider Figure 1(a). The pair $(x, y)$ is ML and the current centroids are $C_{i}, i=1, \ldots, 6$. The distances are shown in the figure. Depending on the values of $R, \delta$, and $\epsilon$, points may be assigned to any of the pairs $\left(C_{1}, C_{2}\right),\left(C_{3}, C_{4}\right)$, or $\left(C_{5}, C_{6}\right)$. Hence, all $K^{2}$ options must be checked for every constraint.

Another issue arises from the fact that the penalty for violated links depends on the distance between the corresponding centroids, but the locations of the instances are not taken into account. Consider the two clustering problems shown in Figure 1(b). In both, the initial centroids are $C_{1}, C_{2}$. One problem includes the ML $\left(x_{1}, y\right)$, while the other includes $\mathrm{ML}\left(x_{2}, y\right)$. Table 1 summarizes the available assignments in each case and the CVQE value. Note that, regardless of $d$ and $f$, both problems always have the same solution, although our intuition says that violating $\left(x_{1}, y\right)$ is "worse" than violating $\left(x_{2}, y\right)$. Furthermore, the corrective action in both cases is the same: move $C_{1}$ along the line connecting it to $C_{2}$, completely ignoring the relative orientation of the offending instance.

\section{The LCVQE Algorithm}

Our modification of CVQE follows. The proposed algorithm minimizes a target function composed of the vector quantization error as well as a penalty for violated constraints, in the style of CVQE. For each constraint assignment, the LCVQE algorithm considers at most two naturally chosen clusters, hence its complexity is independent of $K$. Informally, violated ML constraints update each centroid toward the opposite instance, hence they are symmetric in instance order. For violated CL constraints, the instance that is farther from the cluster 
Table 1. CVQE values for Figure 1

\begin{tabular}{|c|c|c|c|c|}
\hline Algorithm & Constraint & $y \in Q_{1}, x_{i} \in Q_{2}$ & $x_{i}, y \in Q_{1}$ & $x_{i}, y \in Q_{2}$ \\
\hline \hline CVQE & $\left(x_{1}, y\right)$ & $R^{2}+R^{2}+f^{2}$ & $R^{2}+d^{2}$ & $R^{2}+d^{2}$ \\
\hline CVQE & $\left(x_{2}, y\right)$ & $R^{2}+R^{2}+f^{2}$ & $R^{2}+d^{2}$ & $R^{2}+d^{2}$ \\
\hline \hline LCVQE & $\left(x_{1}, y\right)$ & $\left(d^{2}+d^{2}\right) / 2$ & $d^{2}$ & $d^{2}$ \\
\hline LCVQE & $\left(x_{2}, y\right)$ & $\left(d^{2}+d^{2}\right) / 2$ & $d^{2}$ & $d^{2}$ \\
\hline
\end{tabular}

centroid is determined and the closest centroid to that instance (other than the current centroid) is moved towards it.

To formalize the algorithm, we define two new functions. $R_{j}(l)$ returns the instance among $s_{1}(l), s_{2}(l)$ whose distance to $C_{j}$ is larger. $\operatorname{MM}(s)$ returns the centroid which is the closest to $s$, other than $C_{M(s)}$. The LCVQE update rule is given by:

$$
\begin{gathered}
C_{j}=\frac{1}{N_{j}}\left\{\sum_{s_{i} \in Q_{j}} s_{i}+\frac{1}{2} \sum_{l=1, g(l)=j}^{r} v(l) \cdot s_{2}(l)+\frac{1}{2} \sum_{l=1, g^{\prime}(l)=j}^{r} v(l) \cdot s_{1}(l)\right. \\
\left.+\sum_{l=r+1, j=\operatorname{MM}\left(R_{M\left(s_{1}(l)\right)}(l)\right)}^{s+r} v(l) \cdot R_{M\left(s_{1}(l)\right)}(l)\right\} \\
N_{j}=\left|Q_{j}\right|+\frac{1}{2} \sum_{l=1, g(l)=j}^{r} v(l)+\frac{1}{2} \sum_{l=1, g^{\prime}(l)=j}^{r} v(l)+\sum_{l=r+1, j=\operatorname{MM}\left(R_{M\left(s_{1}(l)\right)}(l)\right)}^{s+r} v(l) .
\end{gathered}
$$

This update rule minimizes the error function:

$$
\begin{aligned}
E_{j} & =\frac{1}{2} \sum_{s_{i} \in Q_{j}} T_{j, 1}+\frac{1}{2} \sum_{l=1, g(l)=j}^{r} T_{j, 2}+\frac{1}{2} \sum_{l=1, g^{\prime}(l)=j}^{r} T_{j, 3} \\
& +\frac{1}{2} \sum_{l=r+1, j=\operatorname{MM}\left(R_{M\left(s_{1}(l)\right)}(l)\right)}^{s+r} T_{j, 4}
\end{aligned}
$$

Here,

$$
\begin{array}{ll}
T_{j, 1}=\left(C_{j}-s_{i}\right)^{2} & T_{j, 2}=\left[\frac{1}{2}\left(C_{j}-s_{2}(l)\right)^{2} \cdot v(l)\right] \\
T_{j, 3}=\left[\frac{1}{2}\left(C_{j}-s_{1}(l)\right)^{2} \cdot v(l)\right] & \left.T_{j, 4}=\left[\left(C_{j}-R_{M\left(s_{1}(l)\right)}(l)\right)\right)^{2} \cdot v(l)\right]
\end{array}
$$

Detailed pseudo-code is in [11. The LCVQE algorithm requires $O(d)$ operations (for $d$ dimensions) in each step because only three possible assignments are checked, regardless of $K$. Hence, this algorithm is faster and efficient for problems having large numbers of constraints or clusters. Another benefit of the algorithm is that the constraints are symmetric and that the centroid that is updated depends on the exact setting of both instances rather than the violating instance alone.

Finally, our algorithm does a better job of handling the example shown in Figure 1(b). Table 1 summarizes the available assignments in each case and the 

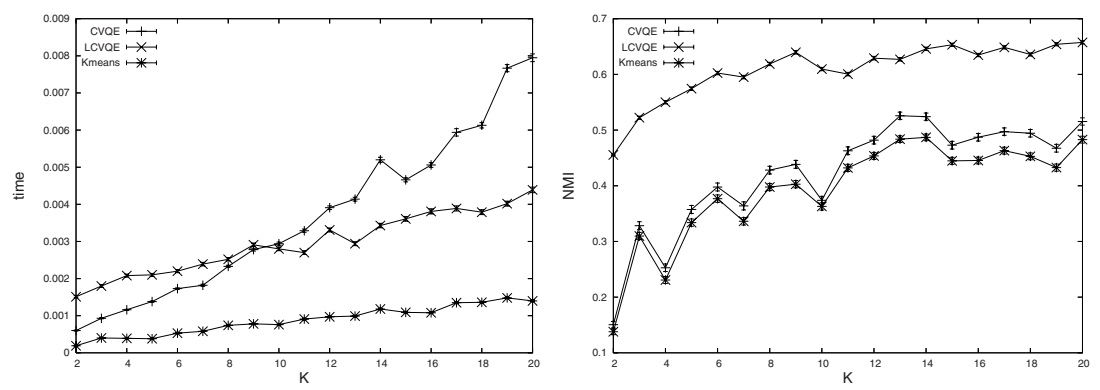

Fig. 2. Performance on Iris

LCVQE values. Assume that the assignment in both problems is $x_{i} \in Q_{2}, y \in Q_{1}$. In the case of $\left(x_{1}, y\right)$, the centroid is updated as follows: $C_{1}=\left(y+\frac{1}{2} x_{1}\right) / 1.5$. In the case of $\left(x_{2}, y\right)$, the centroid is updated as follows: $C_{1}=\left(y+\frac{1}{2} x_{2}\right) / 1.5$, which is intuitively better (because the centroid is moving toward the mean of the instances rather then toward the other centroid).

\section{Experiments}

We first compare the performance of LCVQE and CVQE on UCI data. We implemented both, as well as $K$-means, in $\mathrm{C}$, and used a $3.6 G H z$ Pentium 4 machine for testing. Times in the plots are all in seconds.

We drew random data pairs to generate constraints. Noise was inserted with probability $p=1 \%$ by changing the labels in a pair to labels drawn uniformly from the set of classes. Afterwards, the labels were compared to generate either an ML or a CL constraint. In each case $25 \mathrm{ML}$ and $25 \mathrm{CL}$ constraints were generated.

We generated the augmented set of transitive and entailed constraints in preprocessing, which did not contribute towards the measured run time of any algorithm. To measure clustering performance, we used NMI [7. Figure 2 shows NMI and run time values averaged over 100 runs over the Iris dataset. Data for the other UCI datasets can be found in [11. Accuracy is increased dramatically for all values of $K$ in the Iris and Glass datasets, and is significantly better for most values of $K$ for Ionosphere, Wine, and Pima, and at par for E-coli and Breast. Note that for the Glass and Wine datasets, CVQE is substantially worse than unconstrained $K$-means, a phenomenon we did not observe for LCVQE.

For run times, the quadratic growth in $K$ is clearly visible for CVQE, whereas LCVQE (and, as expected, $K$-means), are linear in $K$. At the same time, there are cases where LCVQE is slower, especially at the low end of $K$. We explore this point below.

Recall that, for each constraint, LCVQE searches over a space that includes just two centroids, whereas CVQE performs the search over all $K$ centroids. This reduction in search space movement has the potential to make each change in 

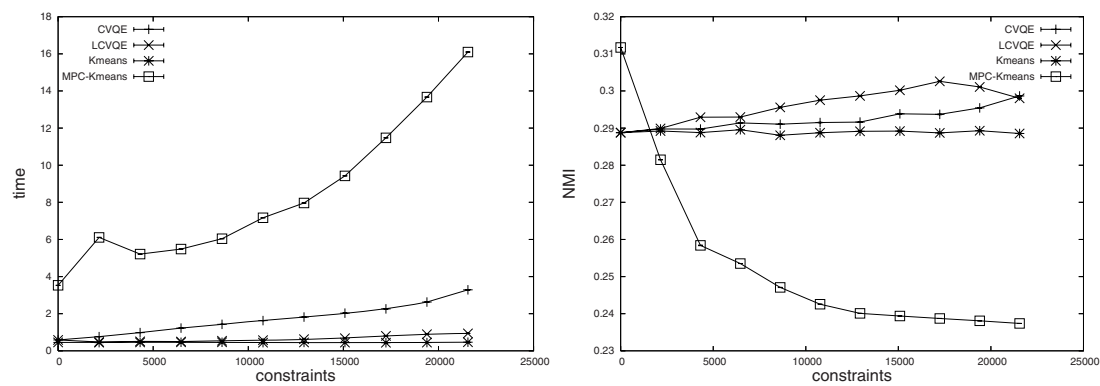

Fig. 3. Performance on tracking data, $k=10$

centroids smaller. We tested this hypothesis, and conclude that that LCVQE does tend to iterate longer than CVQE, which explains the longer run times for low values of $K$. See 11 for details.

We also examined the dependence of run time on the size of the input. Here we added to the mix the $M P C$-Kmeans algorithm [12, which is an efficient hybrid of the distance-learning and constraint-satisfaction approaches 1 . Results are omitted due to lack of space, and can be found in [11. They show that the $K$ means variants are linear in the number of constraints, whereas $M P C$ - Kmeans is super-linear. Here, too, the entailed constraints were generated by the wrapper script, and $M P C-K$ means was instructed not to run its own entailment code.

Our final experiment tests performance on surveillance data from a realistic arena. In it, ten uncontrolled agents move in a 2-D space and interact among themselves and with the surroundings. Each minute, a snapshot is taken, and the location and true identity of each agent are recorded. We generate raw data that corresponds to the locations of agents, and ML constraints between each agent's location and its location at the previous time step. In a sense, this is a version of the famous GPS lane finding data but with greater freedom of movement for the agents. Noise was added as above. Here we did not augment the constraint set (nor did $M P C$-Kmeans run its own augmentation routine). Because of the long chains in the ML graph, the transitive closure becomes huge and mostly uninformative. See Figure 3. We observe that LCVQE produces better or equivalent results to CVQE, while running much faster. In particular, the performance of $M P C-K$ means degrades very quickly when the number of constraints increases. We can speculate that this could be the effect of noise. Another possible reason is the absence of the connected-component heuristic published work on $M P C-K$ means did not explore any of these scenarios.

\section{Conclusion}

Having emerged as a new technology a few short years ago, constrained clustering methods are now transitioning to the status of established practice.

\footnotetext{
${ }^{1}$ In terms of NMI, it outperforms LCVQE on the small UCI datasets.
} 
Consequently, the question of constraint acquisition is increasing in importance. Without automatic constraint generation, plugging in a constrained method in place of a traditional unsupervised method is impossible. We hypothesize that, in many realistic scenarios, the graphs of generated constraints will look very different from the label-based constraint sets that are traditionally used to evaluate new algorithms. In particular, the graphs of ML constraints are likely to contain long chains rather than (dense or sparse) cliques. An interesting avenue of research is to explore the effect of graph structure and noise on the qualities and desired properties of constrained clustering algorithms, in the spirit of Davidson et al. [13. We explore this issue in a forthcoming paper.

In this light, we discuss a real-world tracking scenario where data points and constraints are plentiful and possibly noisy. This notion alone breaks the consistency assumption central to many of the existing constrained clustering algorithms, resulting in poor performance. We propose a scalable and robust

algorithm, based on the CVQE framework, capable of operating in this kind of environment. We show extensive experimental results that shed light on the relative performance of both algorithms and compare them to a distance-learning algorithm.

\section{Acknowledgments}

We thank Ian Davidson for helpful comments and discussion.

\section{References}

1. Wagstaff, K., Cardie, C., Rogers, S., Schroedl, S.: Constrained $k$-means clustering with background knowledge. In: Brodley, C., Danyluk, A. (eds.) Proceeding of the 17th International Conference on Machine Learning, Morgan Kaufmann, San Francisco (2001)

2. Lin, W.-H., Hauptmann, A.: Structuring continuous video recordings of everyday life using time-constrained clustering. In: IS\&T/SPIE Symposium on Electronic Imaging, San Jose, CA (January 2006)

3. Hertz, T., Shental, N., Bar-Hillel, A., Weinshall, D.: Enhancing image and video retrieval: Learning via equivalence constraints. In: Proc. of IEEE Conference on Computer Vision and Pattern Recognition, IEEE Computer Society Press, Los Alamitos (2003)

4. Davidson, I., Ravi, S.S.: Clustering with constraints: Feasibility issues and the $k$-means algorithm. In: 5th SIAM Data Mining Conference (2005)

5. Yu, S.X., Shi, J.: Grouping with directed relationships. In: Figueiredo, M., Zerubia, J., Jain, A.K. (eds.) EMMCVPR 2001. LNCS, vol. 2134, Springer, Heidelberg (2001)

6. Cohn, D., Caruana, R., McCallum, A.: Semi-supervised clustering with user feedback. Technical report, Cornell University, TR2003-1892 (2003)

7. Basu, S., Bilenko, M., Mooney, R.J.: A probabilistic framework for semi-supervised clustering. In: Kim, W., Kohavi, R., Gehrke, J., DuMouchel, W. (eds.) Proceedings of the Tenth ACM SIGKDD International Conference on Knowledge Discovery and Data Mining, Seattle, WA, pp. 59-68. ACM Press, New York (2004) 
8. Basu, S., Davidson, I.: Clustering with constraints: Theory and practice. In: Online Proceedings of a KDD tutorial (2006),

http://www.ai.sri.com/ basu/kdd-tutorial-2006/

9. Davidson, I., Ravi, S.S.: Identifying and generating easy sets of constraints for clustering. In: AAAI, AAAI Press (2006)

10. Davidson, I., Wagstaff, K., Basu, S.: Measuring constraint-set utility for partitional clustering algorithms. In: Fürnkranz, J., Scheffer, T., Spiliopoulou, M. (eds.) PKDD 2006. LNCS (LNAI), vol. 4213, pp. 115-126. Springer, Heidelberg (2006)

11. Pelleg, D., Baras, D.: k-means with large and noisy constraint sets. Technical Report H-0253, IBM (2007)

12. Bilenko, M., Basu, S., Mooney, R.J.: Integrating constraints and metric learning in semi-supervised clustering. In: Brodley, C.E. (ed.) ICML, ACM, New York (2004)

13. Davidson, I., Ravi, S.S.: Intractability and clustering with constraints. In: ICML (2007) 